\title{
Hybrid Electric Vehicle Traffic Flow Image Restoration using Blind Deconvolution Algorithm
}

\author{
Qingsheng Shi*, Ke Lu and Shijie Zhang \\ College of Electrical Engineering, Henan University of technology, Zhengzhou 450001, China \\ ${ }^{*}$ Corresponding author
}

\begin{abstract}
With the characteristics of multi-source, time-varying and fuzzy, traffic decision information needed by hybrid vehicle energy management system has become the major bottleneck to restrict hybrid electric vehicle's energy optimization. The first step to obtain valid traffic information is image processing of traffic flow. In practice, blurring and noise often has an adverse effect on the performance of image processing. Blind deconvolution algorithm was studied in the image restoration of hybrid electric vehicle traffic flow. Simulation results show the validity of proposed algorithm.
\end{abstract}

Keywords-hybrid electric vehicle; energy management; blind deconvolution; image deblurring

\section{INTRODUCTION}

The shortage of energy and air pollution are two major problems facing the world, vigorously developing hybrid vehicles and other new energy vehicles is the effective way to alleviate the problem ${ }^{[1,2]}$. In Europe, hybrid electric vehicles have developed nearly a century. While the hybrid vehicle research of China began in the last century 90's ${ }^{[3]}$. The practical significance of developing hybrid vehicles are as follows: First, it can greatly reduce the excessive dependence on oil in the automotive industry, if mixed on the scale of the power car to car of the annual production capacity of $50 \%$, oil consumption will be reduced by more than $14 \%{ }^{[4]}$; Second, compared to traditional cars, using electric energy in hybrid vehicles can significantly reduce exhaust emissions and reduce air pollution; Third, hybrid vehicles can be charged using valley electricity at night, which would be conducive to the peak shifting and valley filling, and improve energy utilization efficiency.

At home and abroad, the popularity of hybrid electric vehicles is still not high. Many factors should be responsible for it. Among them, optimization of energy management system is particularly prominent. Several control strategies of energy management have been proposed yet, such as logic control, fuzzy control and dynamic control, etc ${ }^{[5-7]}$. To obtain global optimal control of energy management system in hybrid electric vehicle, traffic decision information is needed. However, traffic information shows the characteristics of multi-source, time varying and fuzzy ${ }^{[8]}$. To obtain valid traffic information, image processing of traffic flow is needed first. In practice, blurring and noise often has an adverse effect on the performance of image processing. There are mainly four methods to image restoration: blind deconvolution algorithm, Lucy-Richardson algorithm, regularized filter and Wiener filter. In the paper, blind deconvolution algorithm was studied in the image processing of hybrid electric vehicle traffic flow.

\section{Hybrid EleCtric Vehicle TrafFic Flow IMAgE} DEBluRring Using Blind DECONVOLUTION AlgORITHM

In practice, the following factors often cause traffic flow blurring image: a) Movement during the image capture process, by the camera or, when long exposure times are used, by the subject; b) Out-of-focus optics, use of a wide-angle lens, atmospheric turbulence, or a short exposure time, which reduces the number of photons captured; c) Scattered light distortion in confocal microscopy.

As shown in Figure $\mathrm{I}$, through a system $\mathrm{H}$ and additive noise $n(x, y)$, the original image $f(x, y)$ would degrade to image $\mathrm{g}(\mathrm{x}, \mathrm{y})$.The degradation process can be mathematically expressed in formula (1):

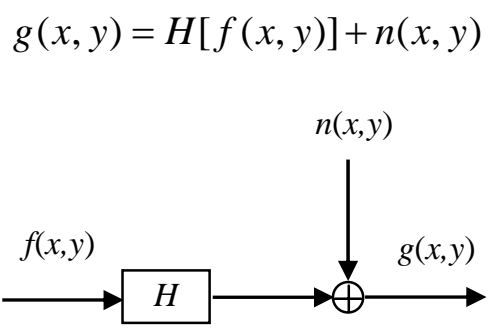

\section{FIGURE I. IMAGE DEGRADATION MODEL}

The goal of image restoration is to seek the methods for estimating the original image on the assumption of some knowledge of $\mathrm{g}, \mathrm{H}$ and $\mathrm{n}$.

Many different methods were attempted to restore our image when we don't explicitly know H. Most of them had very little success. The reasons will be explained as we explain the general approach we used. The methods for estimating hare known as blind deconvolution because inverse filtering is being performed without knowledge of our blurring function.

\section{A. Preliminary of Blind Deconvolution Algorithm}

Blind deconvolution is a deconvolution technique that permits recovery of the target scene from a single or set of "blurred" images in the presence of a poorly determined or unknown point spread function (PSF)[9]. The PSF describes the response of an imaging system to a point source or point 
object. The PSF in many contexts can be thought of as the extended blob in an image that represents an unresolved object. In functional terms it is the spatial domain version of the transfer function of the imaging system. It is a useful concept in Fourier optics, astronomical imaging, medical imaging, electron microscopy and other imaging techniques such as 3D microscopy (like in confocal laser scanning microscopy) and fluorescence microscopy. The degree of spreading (blurring) of the point object is a measure for the quality of an imaging system.

Regular linear and non-linear deconvolution techniques utilize a known PSF. For blind deconvolution, the PSF is estimated from the image or image set, allowing the deconvolution to be performed. Researchers have been studying blind deconvolution methods for several decades, and have approached the problem from different directions. Blind deconvolution can be performed iteratively, whereby each iteration improves the estimation of the PSF and the scene, or non-iteratively, where one application of the algorithm, based on exterior information, extracts the PSF. Iterative methods include maximum a posteriori estimation and expectationmaximization algorithms. A good estimate of the PSF is helpful for quicker convergence but not necessary.

\section{B. Procedure of Traffic Image Deblurring using Blind Deconvolution Algorithm}

The procedure of traffic image deblurring using blind deconvolution algorithm is as follows, as shown in Figure II:

Step1: Traffic flow blurred image capture. Due to camera motion or lack of focus, a real-life traffic flow image that could be blurred. In the paper, blurred traffic flow image can be obtained by convolving a Gaussian filter with the true image.

Step2: Restore the blurred traffic flow image using PSFs of various sizes of the PSF. To determine the size of the PSF, examine the blurred image and measure the width of a blur (in pixels) around an obviously sharp object. Because the size of the PSF is more important than the values it contains, you can typically specify an array of 1's as the initial PSF. If all PSFs are used, go to Step3; others, rerun Step2.

Step3: Improving the restoration. The ringing in the restored image occurs along the areas of sharp intensity contrast in the image and along the image borders. Therefore, the ringing effect should be reduced by specifying a weighting function. Blind deconvolution algorithm weights each pixel according to the WEIGHT array while restoring the image and the PSF.We start by finding the "sharp" pixels using the edge function. Then, imdilate and pass is used in a structuring elements to widen the area. And then, the pixels closed to the borders are assigned the value 0 .

Step4: output the deblurred image.

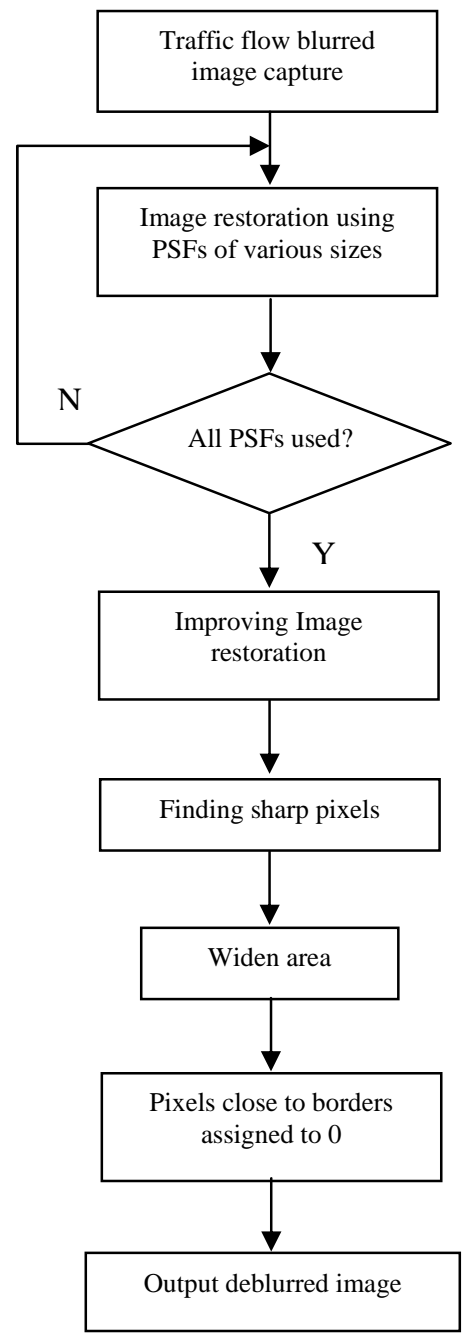

FIGURE II. TRAFFIC FLOW IMAGE DEBLURING PROCESS

\section{SiMULATION RESULTS AND ANALYSIS}

In order to evaluate the performance of blind deconvolution algorithm in traffic flow image deblurring, a blurred traffic flow image is adopted, as shown in Figure III.

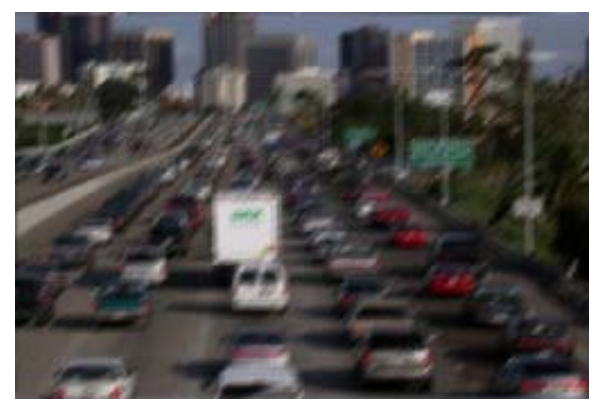

FIGURE III. BLURRED TRAFFIC FLOW IMAGE

During the simulation, the blurred image is obtained by convolving a Gaussian filter with the true image. 
Parameters setting for simulation are as follows: the value for size of filter is [3 3], the value for standard deviation sigma is 0.5 , the number of iterations is set to 30 .

The deblurred traffic flow image is shown in Figure IV.Compared to blurred image in Figure III, using blind deconvolution algorithm has effectively deblurred the traffic flow image. The corresponding PSF is shown in Figure V. It is seen from Figure $\mathrm{V}$, there is no edge-related ringing.

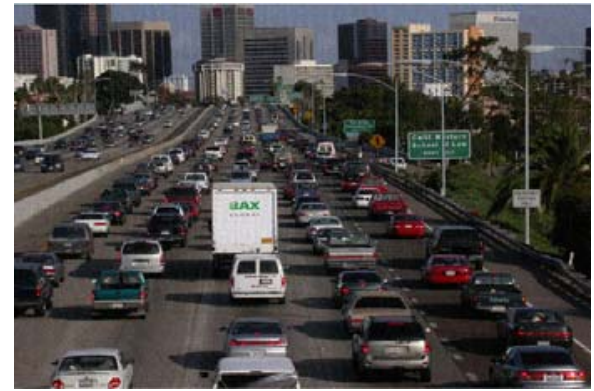

FIGURE IV. DEBLURRED TRAFFIC FLOW IMAGE

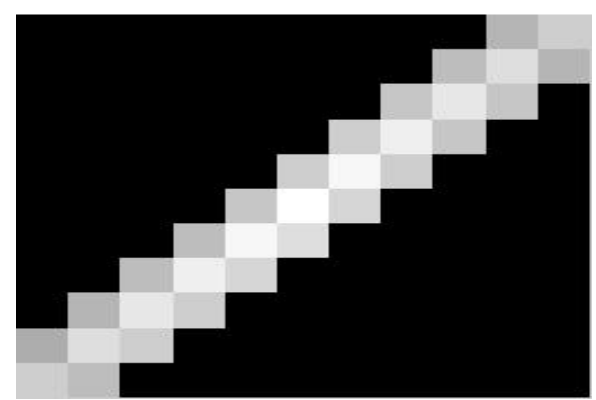

FIGURE V. NEWLY RECONSTRUCTED PSF

\section{CONCLUSIONS}

In the paper, hybrid electric vehicle traffic flow image deblurring is studied using blind deconvolution algorithm. Blurring and noise often has an adverse effect on the performance of image processing, and further influence the performance of energy management of hybrid vehicle. Simulation results show that, using blind deconvolution algorithm can effectively deblur the traffic flow image.

\section{ACKNOWLEDGMENT}

This work is supported by National Natural Science Foundation (NNCF) of China under Grant 61403124 and innovation talent cultivation project of Henan University of Technology 2012CXRC13.

\section{REFERENCES}

[1] C.C. Chan, The state of the art of electric and hybrid vehicles, Proceedings of the IEEE, vol. 90, issue 2, pp. 247 - 275, Feb. 2002

[2] Alireza Khaligh, Zhihao Li. Battery, Ultracapacitor, Fuel Cell, and Hybrid Energy Storage Systems for Electric, Hybrid Electric, Fuel Cell, and Plug-In Hybrid Electric Vehicles: State of the Art, IEEE Transactions on Vehicular Technology, 2010, 59(6): 2806-2814

[3] China 2012 crude oil imports rose $6.8 \%$, http://www.china5e.com/show.php?contentid= 262839
[4] Chen Qingquan, Sun Fengchun, Zhu Jiaguang. Modern electric vehicle technology [M]. Beijing: Beijing institute of technology press,2002.

[5] Lee H D,ul S K Fuzzy logic based torque control strategy for parallel type hybrid electric vehicle[J],EEE Transactions on Industrial Electronics,1998,45(4):25-632

[6] Ehsani M,Gao Yimin,Butler K L Application of electrically peaking hybrid (ELPH) propulsion system to a full-size passenger car with simulated design verification [J], IEEE Transactions on Vehicular Technology, 1999, 48(6):1779-1787

[7] Lin C C,Peng H,Grizzle J W. Power management strategy for a parallel hybrid electric truck[J]. IEEE Transactions on Control Systems Technology, 2003, 11(6):839-849

[8] W. Andreas, W. Shangbo, G. H. Bruck, et al. Traffic congestion estimation service exploiting mobile assisted positioning schemes in GSM networks [J]. Procedia Earth and Planetary Science, 2009,1(1):1385-1392

[9] E. Lam; J.W. Goodman (2000). "Iterative statistical approach to blind image deconvolution". Journal of the Optical Society of America A 17 (7): 1177-1184 